\title{
MicroRNA-124 regulates TRAF6 expression and functions as an independent prognostic factor in colorectal cancer
}

\author{
CHEN WEI ${ }^{1,2^{*}}$, LIU LEI $^{2 *}$, HUANG HUI $^{2}$ and ZHANG TAO ${ }^{2}$ \\ ${ }^{1}$ Key Laboratory for Molecular Diagnosis of Hubei Province; \\ ${ }^{2}$ Department of Gastrointestinal Surgery, Central Hospital of Wuhan, Tongji Medical College, \\ Huazhong University of Science and Technology, Wuhan, Hubei 430014, P.R. China
}

Received June 9, 2018; Accepted April 18, 2019

DOI: $10.3892 / \mathrm{ol} .2019 .10358$

\begin{abstract}
An increasing number of studies have confirmed that miR-124 exhibits a suppressive role in glioblastoma, cervical cancer and breast cancer; however, the function of miR-124 in colorectal cancer (CRC) has not been completely elucidated. In the present study, miR-124 expression was confirmed by reverse transcription-quantitative PCR in 80 colorectal tissues and para-cancerous tissues. The influence of altered miR-124 expression was analyzed by statistical approaches including Cox multivariate regression analysis and the Kaplan-Meier method, and the target genes of miR-124 were confirmed by luciferase reporter assays. Immunohistochemical techniques were also performed in order to measure the expression levels of target proteins. miR-124 expression was observed to be decreased in colorectal tissue samples, and this phenomenon was correlated with adverse clinical indicators and poor patient survival time. Luciferase reporter assays indicated that miR-124 directly regulated TNF receptor associated factor 6 (TRAF6) 3'-untranslated region (UTR). Hence, it was proposed that miR-124 dysregulation may negatively influence the expression of TRAF6 and therefore serve as a biomarker of epithelial-mesenchymal transition in CRC tissues. In summary, the present study demonstrated that miR-124 regulates the expression of TRAF6, and may potentially function as an independent prognostic factor and therapeutic target in patients with CRC.
\end{abstract}

Correspondence to: Professor Zhang Tao, Department of Gastrointestinal Surgery, Central Hospital of Wuhan, Tongji Medical College, Huazhong University of Science and Technology, Nanjing Road, Wuhan, Hubei 430014, P.R. China

E-mail: zhangtaogi@163.com

${ }^{*}$ Contributed equally

Abbreviations: TRAF6, tumor necrosis factor receptor-associated factor 6; EMT, epithelial-mesenchymal transition; CRC, colorectal cancer

Key words: microRNA-124, CRC, prognosis, TRAF6, metastasis

\section{Introduction}

Colorectal cancer (CRC) is a common malignant disease which is a profound public health burden (1). The risk of CRC incidence and mortality in China is enhanced by tobacco smoking, alcohol consumption, obesity, physical inactivity, low fruit and vegetable consumption and the high intake of red and processed meat (2). A recent epidemiological study reported that the incidence of $\mathrm{CRC}$ has increased over the past few decades (3). Despite improvements in the available treatments, the 5-year survival rate in China remains low, which is attributed to the majority of patients being diagnosed at a late disease stage (4). Numerous cases of CRC present with metastasis of the peripheral lymph nodes and organs (5).

Epithelial-mesenchymal transition (EMT) is characterized by the loss of epithelial cell characteristics through the transition to a more malignant, mesenchymal cell phenotype (6). The loss of cell polarity combined with the breakdown of tight intercellular junctions results in a high risk of tumor metastasis (7). Recent evidence has demonstrated that aberrant EMT activation in CRC is closely associated with carcinogenesis and tumor progression $(8,9)$. Following the onset of EMT, E-cadherin is translated into $\mathrm{N}$-cadherin, which is expressed in interstitial cells. Consequently the deletion and downregulation of E-cadherin is considered to be an important marker of EMT (10). Vimentin is also an important mesenchymal cell marker of EMT, which serves an important role in maintaining interstitial cell characteristics. Studies have confirmed that the increased expression of Vimentin is also associated with tumor invasion and metastasis (10).

In a previous study, tumor necrosis factor receptor-associated factor 6 (TRAF6) was identified as a prognostic factor in CRC (11). The prominent expression of TRAF6 has also been observed in other human malignancies, such as lung and gastric cancer, nasopharyngeal carcinoma and breast cancer (12-15). TRAF6 belongs to the TRAF family, and acts as an adaptor in the signaling of channels induced by the TNFR. An increasing number of studies have indicated that TRAF6 promotes oncogenesis by attenuating cell apoptosis and accelerating proliferation and invasion in tumor lesions $(14,16)$. Notably, TRAF6 is a target gene of miR-124 and the latter was reported to be involved in EMT in multiple 
malignant diseases $(17,18)$. However, the role and relationship between miR-124 and TRAF6 in CRC remains unclear.

In the present study miR-124 was downregulated in CRC tissues, and a decrease in the level of miR-124 was associated with the specific adverse features of CRC, indicating an increased risk of poor overall survival. The data also demonstrated that TRAF6 was a target gene for miR-124 and is potentially involved in EMT. Taken together these results suggest that miR-124 may exhibit a tumor-suppressive role by regulating TRAF6 expression in CRC.

\section{Materials and methods}

Patients and follow up. A total of 80 patients with a median age of 64.5 years (range, 40-72) who underwent surgery at the first presentation of CRC at the Central Hospital of Wuhan between January 2008 and December 2012 were selected for inclusion in the present study. None of the patients had received additional treatment prior to surgical intervention. Patients' clinical data are presented in Table I. CRC was classified according to the American Joint Committee on Cancer (19) staging system. The endpoint of this research was described as overall survival. All patients provided written informed consent to participate prior to surgery. Ethical approval was given by the medical ethics committee of the Central Hospital of Wuhan Tongji Medical College, Huazhong University of Science and Technology. All procedures performed in this study involving human participants were conducted in accordance with Chinese ethical standards and the 2008 Declaration of Helsinki.

$R N A$ extraction and reverse transcription-quantitative $P C R$ (RT-qPCR). Total RNA was extracted from cells and tissues using TRIzol ${ }^{\circledR}$ reagent (Wuhan Guge Biotechnology Co., Ltd.), according to the manufacturer's protocol. The Thermo Nano Drop 2000 (Nano Drop Technologies; Thermo Fisher Scientific, Inc.) was used to determine the concentration and purity of the RNA. The primer sequences were as follows: Hsa-miR-124-3p forward, 5'-ACACTCCAGCTGGGTAAGGCACGCGGTG-3', and reverse, 5'-CTCAACTGGTGTCGTGGAGTCGGCAAT TCAGTTGAGGGCATTCA-3'. U6 forward, 5'-CTCGCT TCGGCAGCACA-3', and reverse, 5'-AAACGCTTCACG AATTTGCGT-3'. RT-qPCR was performed using the iTaq Universal SYBR ${ }^{\circledR}$ Green Supermix and CFX96 real-time PCR system (Bio-Rad Laboratories, Inc.). The primers are listed in Table II. RT-qPCR was performed in line with a commonly used method described previously (20). Primers for U6 and miR-124 were synthesized and purified by Guangzhou Ribo Bio Co. Ltd. U6 was used as the endogenous control. Relative fold expressions were calculated with the comparative quantification cycle $\left(2^{-\Delta \Delta \mathrm{Cq}}\right)$ method (21). The expression levels of miR-124 were compared between cancerous and para-cancerous tissues, with the cancer/para-cancerous ratio as the ordinate; a ratio $>1$ indicated a high miR-124 expression level, and a ratio $<1$ indicated that miRNA-124 expression was downregulated.

Immunohistochemistry (IHC). The pathological examination of samples was performed at the Central Hospital of Wuhan, Tongji Medical College, Huazhong University of Science and
Technology using a two-step method (22). Tissues were fixed using 4\% paraformaldehyde at room temperature for $24 \mathrm{~h}$. Paraffin-embedded tissue sections were cut into $5 \mu \mathrm{m}$-thick sections and deparaffinized and rehydrated with xylene and a graded alcohol series $(100,95,85$ and $75 \%)$ at room temperature. Sections were washed with PBS three times $(3$ min each time). Antigen retrieval was performed with $0.01 \mathrm{M}$ citrate buffer at $98^{\circ} \mathrm{C}$ for $10 \mathrm{~min}$ and cooled to $37^{\circ} \mathrm{C}$. The sections were washed three times with PBS for $3 \mathrm{~min}$. Subsequently, $50 \mu \mathrm{l}$ of $3 \%$ hydrogen dioxide solution was added to each section and incubated at room temperature for $10 \mathrm{~min}$, followed by washing with PBS. For heat-induced antigen retrieval, the sections were treated with EDTA buffer, autoclaved and returned to room temperature. Antibodies against the target proteins were as follows: TRAF6 (1:50; cat no. ab58369; Abcam), E-cadherin (1:100; cat. no. MA106302; Thermo Fisher Scientific, Inc.) and Vimentin (1:100; cat. no. MA106908; Thermo Fisher Scientific, Inc.). IHC staining was performed according to the following standard procedure (23) to evaluate TRAF6, E-cadherin and Vimentin protein expression in CRC tissues.

Cell cultures and transfection. The human colorectal cancer cell line SW480 was obtained from the China Center for Type Culture Collection. Cells were cultured in DMEM supplemented with 10\% FBS (Sigma-Aldrich, Merck KGaA), $100 \mathrm{U} / \mathrm{ml}$ penicillin (HyClone; GE Health care Life Sciences), and $100 \mu \mathrm{g} / \mathrm{ml}$ streptomycin (HyClone; GE Health care Life Sciences). miR-124 mimics and inhibitor were purchased from Gene Copoeia, Inc. and transfected with a concentration of $50 \mathrm{nM} /$ well into cells with Lipofectamine ${ }^{\circledR} 2000$ (Invitrogen; Thermo Fisher Scientific, Inc.) according to the manufacturer's protocol. The cells were harvested 2 days after transfection for further experimentation.

Western blotting. RIPA lysis buffer (Wuhan Sanying Biotechnology) was used to extract total cell protein. Proteins (40 $\mu \mathrm{g}$ ) were then separated by $10 \%$ SDS-PAGE (Wuhan Guge Biotechnology Co., Ltd.) and transferred to PVDF membranes. After blocking with 5\% non-fat milk for $1 \mathrm{~h}$ at room temperature, the membranes were incubated with the indicated antibodies overnight at $4^{\circ} \mathrm{C}$. The membranes were incubated with anti-TRAF6 (1:1,000; cat. no. 66498-1-Ig; Protein Tech Group, Inc.) or GAPDH (1:1,000; cat. no. 10494-1-AP; Protein Tech Group, Inc.) antibodies overnight at $4^{\circ} \mathrm{C}$ and subsequently incubated with matched secondary antibodies (Wuhan Guge Biotechnology Co., Ltd.). An enhanced chemiluminescence detection system (Wuhan Guge Biotechnology Co., Ltd.) was used to detect the bands. ImageJ software (National Institutes of Health Bethesda, MD, USA) was used to measure the band density. GAPDH was used as a loading control.

Luciferase reporter assays. The relationship between the expression level of miR-124 and TRAF6 in SW480 cells was determined by luciferase reporter assay according to the protocol of a previous study (21). After analyzing the biochemical information database, miR-124 was revealed to act on the 3'-untranslated region (UTR) of TRAF6 and influence its biological activity. Briefly, the wild-type (WT) or mutant (Mt) segments of the TRAF6 3 egment were amplified and cloned into luciferase reporter plasmids (Sino Geno Max 
Table I. Association between miR-124 expression level and the clinicopathological parameters of patients with colorectal cancer.

\begin{tabular}{|c|c|c|c|c|}
\hline \multirow[b]{2}{*}{ Characteristics } & \multirow[b]{2}{*}{ Patients, $n=80$} & \multicolumn{2}{|c|}{$\begin{array}{l}\text { miR-124 } \\
\text { expression }\end{array}$} & \multirow[b]{2}{*}{ P-value } \\
\hline & & Low & High & \\
\hline Age, years & & & & 0.794 \\
\hline$>65$ & 40 & 30 & 10 & \\
\hline$\leq 65$ & 40 & 31 & 9 & \\
\hline Sex & & & & 0.657 \\
\hline Male & 47 & 35 & 12 & \\
\hline Female & 33 & 26 & 7 & \\
\hline Size, $\mathrm{cm}$ & & & & 0.273 \\
\hline$>5$ & 34 & 28 & 6 & \\
\hline$\leq 5$ & 46 & 33 & 13 & \\
\hline Tumor grade & & & & $0.046^{\mathrm{a}}$ \\
\hline Well + moderate & 57 & 40 & 17 & \\
\hline Poor & 23 & 21 & 2 & \\
\hline Lymph node status & & & & $0.017^{\mathrm{a}}$ \\
\hline$<1$ & 52 & 44 & 8 & \\
\hline$\geq 1$ & 28 & 17 & 11 & \\
\hline Distant metastasis & & & & 0.529 \\
\hline Yes & 7 & 6 & 1 & \\
\hline No & 73 & 55 & 18 & \\
\hline TNM & & & & 0.597 \\
\hline $\mathrm{I}+\mathrm{II}$ & 55 & 41 & 14 & \\
\hline III + IV & 25 & 20 & 5 & \\
\hline
\end{tabular}

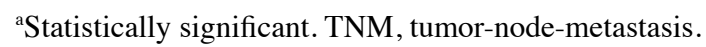

Co., Ltd, Beijing, China) for subsequent experiments. Cells were seeded in 6 -well culture plates at a density of $1 \times 10^{5} / \mathrm{ml}$. Following a 24-h incubation, when the cell confluence had reached $70 \%$, cells were co-transfected with the reporter plasmids and miR-124 mimics or inhibitors at a concentration of $4 \mu \mathrm{g}$ per/well using Lipofectamine ${ }^{\circledR} 2000$ (Invitrogen; Thermo Fisher Scientific, Inc.). At $48 \mathrm{~h}$ post-transfection, the Dual-Luciferase ${ }^{\circledR}$ Reporter Assay kit (Promega Corporation) with a Renilla luciferase normalization control was used to detect the fluorescence intensity of the cells, according to the manufacturers' protocol. All the procedures were repeated $\geq 3$ times.

Statistical analyses. Non-parametric analysis between groups was performed using the Mann-Whitney U test, and the Spearman's rank correlation coefficient was employed to examine the relationship between miR-124 expression level and clinicopathological parameters. Categorical variables were analyzed using $\chi^{2}$ tests for univariate analysis. A paired Student's t-test was performed to analyze the paired data. ANOVA and the Bonferroni correction post-hoc test were applied in multiple comparison analysis. The Kaplan-Meier method was used to draw survival curves, and the differences were verified using the log-rank test. Whether a factor was an independent predictor of CRC prognosis was determined by Cox-multivariate analyses. Statistical analyses were performed using SPSS software (version 19.0; IBM Corp.). $\mathrm{P}<0.05$ was considered to indicate a statistically significant difference.

\section{Results}

miR-124 expression level is significantly lower in tumor tissues than in adjacent para-cancerous tissues. To examine the expression level of miR-124 in colorectal cancer tissues, RT-qPCR was performed to compare expression levels in 80 pairs of CRC and adjacent non-cancerous tissues. As illustrated in Fig. 1A, miR-124 expression level was significantly down regulated in cancer tissues compared with neighboring para-cancerous tissues $(\mathrm{P}<0.05)$.

Relationship between miR-124 expression and clinical pathological parameters. Recent studies have suggested that clinical features such as tumor size, pathological grade, TNM stage and lymphatic metastasis are closely associated with patient prognosis $(24,25)$. On the basis of the data displayed in Table I, patients that presented with a low miR-124 expression level exhibited poor pathological differentiation $(\mathrm{P}=0.046)$ and an increased risk of lymph node metastasis $(\mathrm{P}=0.017)$. No significant associations were identified between miR-124 expression and sex $(\mathrm{P}=0657)$, age $(\mathrm{P}=0.794)$, tumor size $(\mathrm{P}=0.273)$, distant metastasis $(\mathrm{P}=0.597)$ or advanced TNM stage $(\mathrm{P}=0.529)$.

Correlation between miR-124 expression level and CRC patient prognosis. The time between the date of surgical resection and mortality or last patient contact was defined as overall survival. Among the 80 patients with CRC, 47 (58.8\%) died during follow-up as a result of their malignancy. CRC patients with an elevated expression level of miR-124 had a significantly longer survival period. Additionally, the prognosis of patients with a low miR-124 expression level was poor compared with patients expressing high levels of miR-124 ( $\mathrm{P}<0.05$; Fig. 1B). Cox regression multivariate analysis revealed that lymph node status (HR, 0.240; 95\% CI, 0.094-0.614; $\mathrm{P}=0.003$ ), tumor metastasis (HR, 0.269; 95\% CI, 0.093-0.780; $\mathrm{P}=0.016$ ), histological grade (HR, 0.474; 95\% CI, 0.243-0.927; $\mathrm{P}=0.029)$ and miR-124 expression (HR, 6.961; 95\% CI, 2.174-22.294; $\mathrm{P}=0.001$ ) were independent predictive factors for the overall survival of patients with CRC (Table III). Furthermore, survival analyses revealed that patients with a low miR-124 expression level had a significantly poorer 5 -year survival $(\mathrm{P}<0.05$; Fig. 1B). Taken together, these data suggested that miR-124 may exhibit a suppressive role in the development of CRC.

Aberrant expression levels of miR-124 and TRAF6 in CRC. TRAF6 expression confers poor prognosis for patients with CRC (11), and has been highlighted as a potential target protein of miR124 in TargetScan (version 7.1; www.targetscan. org) following bioinformatics analysis. To determine whether miR-124 functionally modulates TRAF6 expression in CRC, miR-124 and TRAF6 expression levels we measured in SW480 cells. Cells transfected with an miR-124 inhibitor displayed 
Table II. Primers for gene expression using reverse transcription-quantitative PCR.

Gene

Primer sequence (5'-3')

hsa-miR-124-3p-RT

hsa-miR-124-3p-S

U6-S

U6-A
CTCAACTGGTGTCGTGGAGTCGGCAATTCAGTTGAGGGCATTCA ACACTCCAGCTGGGTAAGGCACGCGGTG CTCGCTTCGGCAGCACA

AACGCTTCACGAATTTGCGT

Hsa, Homo sapiens; miR, microRNA; RT, reverse-transcription.

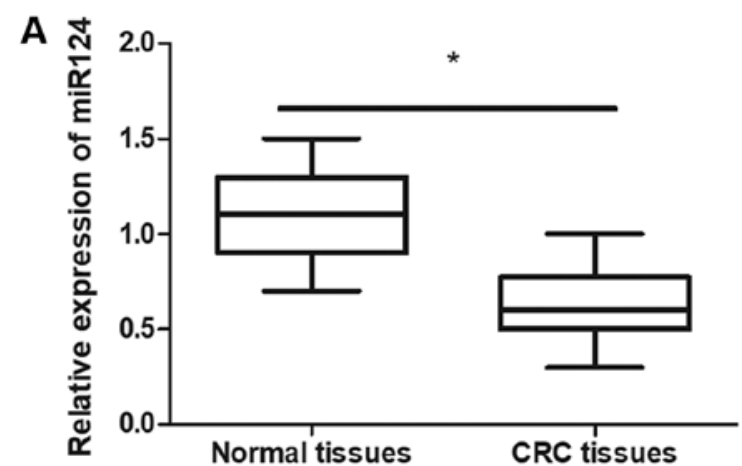

B

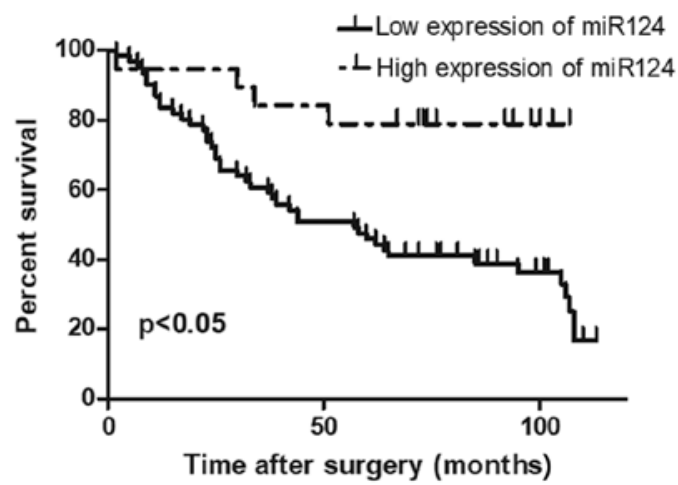

Figure 1. Status and prognostic value of miR-124 expression in CRC. (A) Expression levels of miR-124 between CRC tissues and normal adjacent tissues $(\mathrm{n}=80)$. ${ }^{*} \mathrm{P}<0.05$, as indicated. (B) Kaplan-Meier analysis for overall survival of patients with CLC according to miR-124 expression. Compared with those with high miR-124 levels $(n=19)$, miR-124 low-expressing patients $(\mathrm{n}=61)$ had significantly reduced overall survival times. CRC, colorectal cancer; miR, microRNA.

lower miR-124 expression levels compared with the inhibitor control group, whereas the expression level of miR-124 was markedly upregulated in the miR124 mimic group (Fig. 2). The results revealed that the transfection of SW480 cells with miR-124 constructs was successful. Further evaluation of TRAF6 expression in SW480 cells revealed that TRAF6 expression levels were markedly increased in cells transfected with the miR-124 inhibitor, but downregulated in the miR124 mimic group (Fig. 3). These findings demonstrated a negative association between the expression levels of miR-124 and TRAF6.

miR-124 post-transcriptionally regulates TRAF6 expression. In our previous study, TRAF6 was highly expressed in CRC tissues, which significantly correlated with Dukes' staging, degree of cell differentiation and lymphatic metastasis (11). The present study further investigated whether TRAF6 was a downstream target of miR-124. As illustrated in Fig. 4A, a putative binding site for miR-124 was identified in the 3-UTR of TRAF6. Subsequently, a luciferase reporter assay was performed to investigate whether miR-124 bound to this specific site. Upregulating the expression of miR-124 significantly reduced the luciferase activity of the WT TRAF6 3'-UTR $(\mathrm{P}<0.05)$ whereas downregulating miR124 expression increased the luciferase activity of the WT 3'-UTR $(\mathrm{P}<0.05)$. However, the altered miR-124 expression level did not substantially impact the luciferase activity of the MutTRAF63'-UTR (P>0.05; Fig. 4B).

miR-124 regulates TRAF6 expression and EMT in CRC tissues. To further confirm the association between miR-124 and TRAF6, and to determine whether these molecules are involved in EMT and tumor invasion, the association between the EMT-related biomarkers E-cadherin and Vimentin and TRAF6 in CRC tissues was analyzed via IHC. As illustrated in Fig. 5, the degree of TRAF6 (Fig. 5A) and Vimentin staining (Fig. 5E) was strong in tumors with low miR-124 expression; however, E-cadherin staining was weak (Fig. 5C). In addition, weak TRAF6 (Fig. 5B) and Vimentin (Fig. 5F) staining was observed in tumors with a high miR-124 expression, whereas E-cadherin staining was strong (Fig. 5D). Spearman's correlation analysis demonstrated that miR-124 expression was significantly negatively correlated with that of TRAF6 ( $\mathrm{r}=-0.402 ; \mathrm{P}<0.001)$ and Vimentin $(\mathrm{r}=-0.514$; $\mathrm{P}<0.001)$, yet positively correlated with E-cadherin expression $(\mathrm{r}=0.721 ; \mathrm{P}<0.001)$ in $\mathrm{CRC}$ tissues. These data indicated that miR-124 affects the metastasis of CRC by modulating EMT.

\section{Discussion}

An increasing number of studies have confirmed that miRNAs are active participants in the development of CRC $(26,27)$. In addition, these small non-coding RNAs are regarded as key regulators of metastasis and EMT in human cancers $(28,29)$. Thus, determining the expression levels of different miRNAs during CRC initiation and progression may provide novel insights into the molecular mechanism of carcinogenesis. Fang et al (30) demonstrated that miRNA 449b inhibits SW1116 colon cancer stem cell proliferation by downregulating $G_{1} / S$-specific cyclin-D1 and transcription factor E2F3 
Table III. Cox multivariate regression analysis of miR-124 expression, age, sex, depth of invasion, grade of differentiation, lymph node status and stage in relation to overall survival in patients with colon cancer.

\begin{tabular}{llccccr}
\hline Variable & \multicolumn{1}{c}{ Comparison } & $\beta$ & SE & HR & 95\%CI & P-value \\
\hline miR124 expression & High vs. Low & 1.940 & 0.594 & 6.961 & $2.174-22.294$ & 0.001 \\
Age & $\leq 65$ vs. $>65$ & 0.298 & 0.360 & 1.347 & $0.665-2.731$ & 0.408 \\
Sex & Male vs. Female & 0.366 & 0.919 & 1.422 & $0.238-8.743$ & 0.690 \\
Size, cm & $\leq 5$ vs. $>5$ & 0.281 & 0.908 & 0.755 & $0.127-4.477$ & 0.757 \\
Tumor metastasis & Positive vs. Negative & 1.312 & 0.542 & 0.269 & $0.093-0.780$ & 0.016 \\
Histologic grade & Poor vs. Well, Moderate & 0.746 & 0.342 & 0.474 & $0.243-0.927$ & 0.029 \\
Lymph node status & Positive vs. Negative & 1.425 & 0.478 & 0.240 & $0.094-0.614$ & 0.003 \\
TNM stage & I+II vs. III+IV & 0.784 & 0.432 & 0.457 & $0.196-1.064$ & 0.069
\end{tabular}

$\beta, \beta$ coefficient; SE, standard error; HR, hazard ratio; CI, confidence interval; TNM, tumor-node-metastasis.
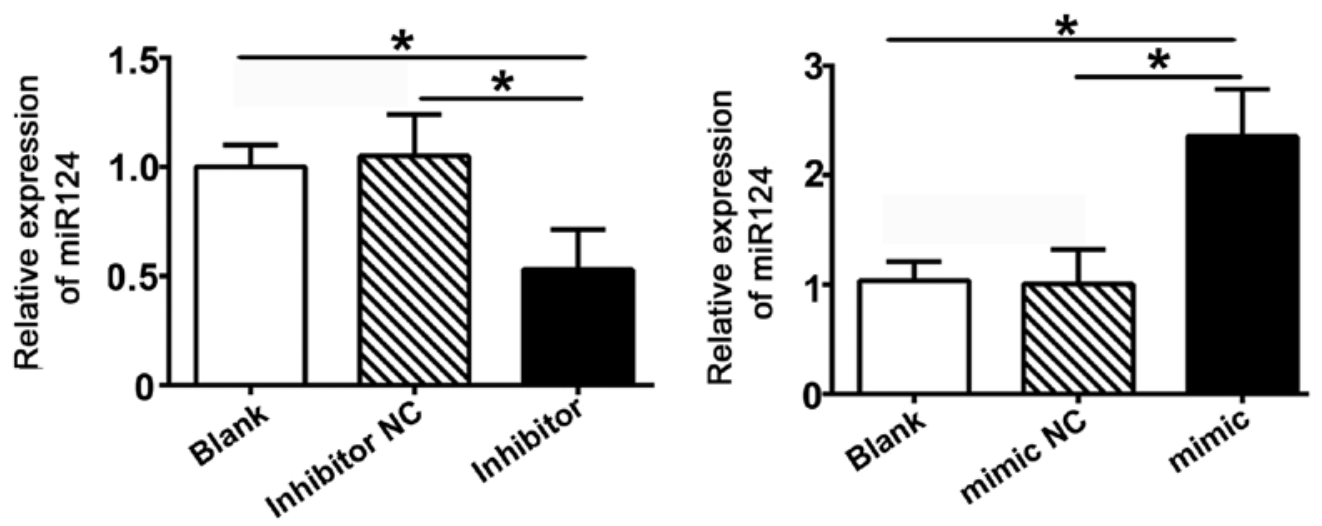

Figure 2. miR-124 expression levels in SW480 cells transfected with miR-124 mimics and inhibitor. ${ }^{*} \mathrm{P}<0.05$, as indicated. miR, microRNA; NC, negative control.
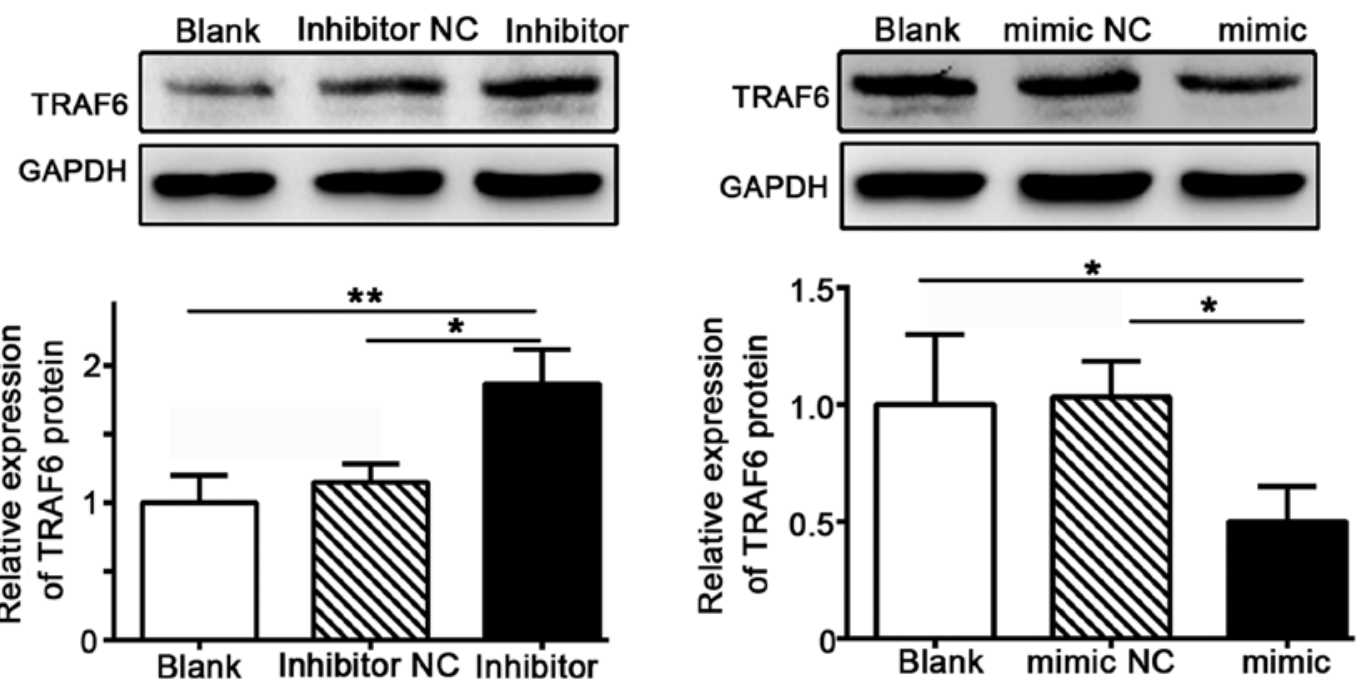

Figure 3. miR-124 suppresses TRAF6 expression in colorectal cancer cells. Cells overexpressing miR-124 showed reduced TRAF6 expression levels. Inhibition of miR-124 in SW480 cells enhanced TRAF6 expression levels. " $\mathrm{P}<0.05$ and ${ }^{* *} \mathrm{P}<0.01$, as indicated. miR, microRNA; NC, negative control; TRAF6, tumor necrosis factor receptor-associated factor 6 .

expression. Liu et al (31) indicated that miR139-3p was an independent prognostic factor of colon cancer, and $\mathrm{He}$ et al (21) revealed that miR-296 attenuated CRC metastasis and EMT by targeting S100A4. Therefore, miRNAs may function as prognostic indicators and potential target biomarkers in the development of novel therapeutics for different types of cancer. 


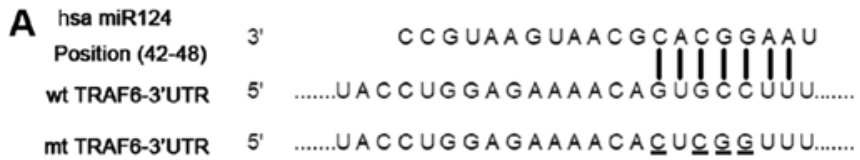

B

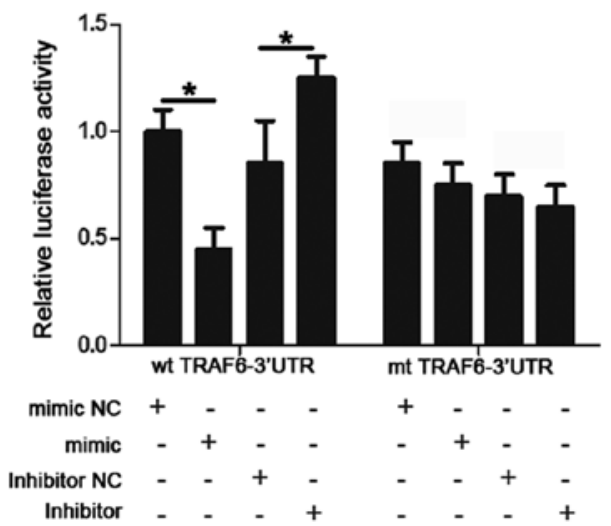

Figure 4. miR-124 binds to the complementary sequence of TRAF6 3'-UTR. (A) The binding sites for miR-124 in the wt and mt 3'-UTR of TRAF6. (B) Over expression of miR-124 decreased, while inhibition of miR-124 increased the luciferase activity of wt 3'-UTR of TRAF6. Alteration of miR-124 had no effect on the luciferase activity of mt 3'-UTR of TRAF6. ${ }^{*} \mathrm{P}<0.05$, as indicated. miR, microRNA; TRAF6, tumor necrosis factor receptor associated factor 6; wt, wild type; mt, mutant; UTR, untranslated region.

In the present study, miR-124 was markedly downregulated in CRC tissues when compared with para-cancerous tissues. In CRC tissues, the miR-124 expression level was significantly correlated with histological grade and lymph node status, which was in agreement with findings from previous studies $(32,33)$. Therefore, it was hypothesized that miR-124 was involved in the development and progression of CRC. In addition, the present study indicated that overall survival time was decreased in CRC patients with a low miR124 expression level, compared with those with a higher expression level $(\mathrm{P}=0.005)$; this provides further evidence that reduced miR-124 expression in CRC may enhance malignant invasion and worsen the prognostic phenotype of this tumor. In a previous in vitro study, miR-124 was proposed to inhibit DNA synthesis and proliferation by reducing ribose-phosphate pyrophosphokinase 1levels in the pentose phosphate pathway (34). Consistent with these data, low miR-124 expression level was directly related to poor prognosis in the present study.

In cancer research, local and/or systemic metastasis represents poor prognosis in patients with CRC (35). A series of reports $(7,36,37)$ confirmed that EMT occurs during CRC progression, which provides cancer cells with invasive and metastatic properties. Therefore, EMT serves a crucial role in cancer metastasis. In a previous study (11), TRAF6 was confirmed to be a weak prognostic marker of CRC and to act on EMT progression. Therefore, the potential association between miR-124 and TRAF6 expression was investigated in the context to EMT. After analyzing IHC-stained colorectal tissue samples, it was discovered that miR-124 expression may be possible negative regulator of EMT in CRC. Strong TRAF6 and Vimentin staining coupled with weak E-cadherin staining was observed in tumors with low miR-124 expression levels. Conversely, high miR-124-expressing tumors presented with

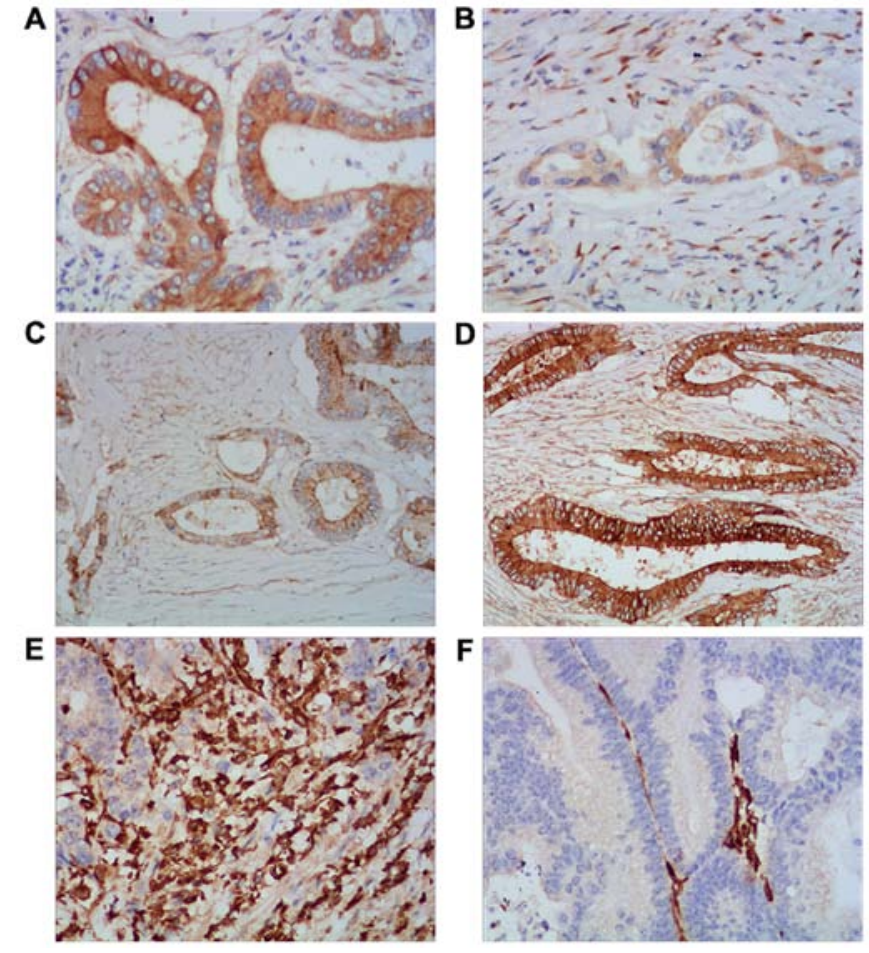

Figure 5. Expression of TRAF6, E-cadherin, and Vimentin in CRC tissues. In representative immunohistochemical staining, miR-124 low-expression tissues exhibited strong (A) TRAF6 and (E) Vimentin staining, and weak (C) E-cadherin staining. However, miR-124 high-expression tissues presented with weak (B) TRAF6 and (F) Vimentin staining, and bright staining of (D) E-cadherin. Magnification, x400. miR, microRNA; TRAF6, tumor necrosis factor receptor associated factor 6; CRC, colorectal cancer.

positive E-cadherin staining but weak Vimentin and TRAF6 staining.

TRAF6 has been identified as an oncogene for its active involvement in malignancy $(38,39)$. Previous research has confirmed that ectopic TRAF6 expression is observed in gastrointestinal tumors $(40,41)$. In the present study, a negative regulatory effect between miR-124 level and TRAF6 expression levels was hypothesized. Strong TRAF6 staining more frequently appeared in CRC tissues with minimal miR-124 expression than in those with high expression levels, and vice versa. In addition, miR-124 directly influenced luciferase reporter activity by interacting with the TRAF6 3'-UTR.

Recently, a study reported that miR-124 inhibited cell invasion and suppressed gastric cancer invasion and metastasis by targeting Snail2 (18). Coincidentally, it was found that high TRAF6 expression levels in CRC tissues were positively correlated with the expression levels of EMT biomarkers. The above data illustrated that miR-124 may serve an important role in EMT in CRC metastasis by regulating the expression of TRAF6. Therefore, the present study suggests that miR-124 and TRAF6 are high-risk indicators for poor patient prognosis, and require further investigation in a larger study cohort.

In summary, the present study demonstrated that miR-124 is poor a prognostic factor in patients with CRC; although miR-124 was shown to influence TRAF6 expression, further evidence is required to determine whether this is by direct or indirect regulation. 


\section{Acknowledgements}

The authors would like to thank Professor Bo Luo and Hanfeng Zhang from the Pathology Department in Tongji Medical College, Huazhong University of Science and Technology for their technical assistance.

\section{Funding}

The present study was supported by the Fund of Health and Family Planning Commission of Wuhan Municipality (grant no. WX17D05) and the Fund of Scientific Researching of the Central Hospital of Wuhan (grant no. YB16A02).

\section{Availability of data and materials}

The datasets generated and/or analyzed during the present study are available from the corresponding author on reasonable request.

\section{Authors' contributions}

CW carried out the molecular genetics studies, participated in sequence alignment and drafted the manuscript. $\mathrm{HH}$ conducted the immunoassays. LL participated in the design of the study and performed the statistical analysis. ZT conceived the study, participated in its design and coordination, and helped to draft the manuscript. All authors have read and approved the final manuscript.

\section{Ethics approval and consent to participate}

All patients provided written informed consent to participate prior to surgery. Ethical approval was given by the medical ethics committee of the Central Hospital of Wuhan Tongji Medical College, Huazhong University of Science and Technology. All procedures performed in this study involving human participants were conducted in accordance with Chinese ethical standards and the 2008 Declaration of Helsinki.

\section{Patient consent for publication}

Written informed consent was obtained from patients for publication of this manuscript and any accompanying images.

\section{Competing interests}

The authors declare that they have no competing interests.

\section{References}

1. Wang YW, Chen HH, Wu MS and Chiu HM; Taiwanese Nationwide Colorectal Cancer Screening Program: Current status and future challenge of population-based organized colorectal cancer screening: Lesson from the first decade of Taiwanese program. J Formos Med Assoc 117: 358-364, 2018.

2. Gu MJ, Huang QC, Bao CZ, Li YJ, Li XQ, Ye D, Ye ZH, Chen K and Wang JB: Attributable causes of colorectal cancer in China. BMC Cancer 18: 38, 2018.

3. Brenner DR, Ruan Y, Shaw E, De P, Heitman SJ and Hilsden RJ: Increasing colorectal cancer incidence trends among younger adults in Canada. Prev Med 105: 345-349, 2017.
4. Zhen YH, Liu XH, Yang Y, Li B, Tang JL, Zeng QX, Hu J, Zeng XN, Zhang L, Wang ZJ, et al: Phase I/II study of adjuvant immunotherapy with sentinel lymph node $\mathrm{T}$ lymphocytes in patients with colorectal cancer. Cancer Immunol Immunother 64: 1083-1093, 2015.

5. Deng Y, Chi P, Lan P, Wang L, Chen W, Cui L, Chen D, Cao J, Wei H, Peng X, et al: Modified FOLFOX6 with or without radiation versus fluorouracil and leucovorin with radiation in neoadjuvant treatment of locally advanced rectal cancer: Initial results of the Chinese FOWARC multicenter, open-label, randomized three-arm phase III trial. J Clin Oncol 34: 3300-3307, 2016.

6. Chen Z, He J, Xing X, Li P, Zhang W, Tong Z, Jing X, Li L, Liu D, Wu Q and Ju H: Mn12Ac inhibits the migration, invasion and epithelial-mesenchymal transition of lung cancer cells by downregulating the Wnt/ $\beta$-catenin and PI3K/AKT signaling pathways. Oncol Lett 16: 3943-3948, 2018.

7. Mao L, Li Y, Zhao J, Li Q, Yang B, Wang Y, Zhu Z, Sun H and Zhai Z: Transforming growth factor- $\beta 1$ contributes to oxaliplatin resistance in colorectal cancer via epithelial to mesenchymal transition. Oncol Lett 14: 647-654, 2017.

8. Jiang M, Xu B, Li X, Shang Y, Chu Y, Wang W, Chen D, Wu N, Hu S, Zhang S, et al: O-GlcNAcylation promotes colorectal cancer metastasis via the miR-101-O-GlcNAc/EZH2 regulatory feedback circuit. Oncogene 38: 301-316, 2019.

9. Hashimoto M, Kobayashi T, Tashiro H, Arihiro K, Kikuchi A and Ohdan H: h-Prune is associated with poor prognosis and epithelial-mesenchymal transition in patients with colorectal liver metastases. Int J Cancer 139: 812-823, 2016.

10. Chang SN, Lee JM, Oh H, Kim U, Ryu B and Park JH: Troglitazone inhibits the migration and invasion of PC-3 human prostate cancer cells by upregulating E-cadherin and glutathione peroxidase 3. Oncol Lett 16: 5482-5488, 2018.

11. Zhang T, Wang $\mathrm{H}$ and Han L: Expression and clinical significance of tumor necrosis factor receptor-associated factor 6 in patients with colon cancer. Iran Red Crescent Med J 18: e23931, 2016.

12. Lou JS, Yan L, Bi CW, Chan GK, Wu QY, Liu YL, Huang Y, Yao P, Du CY, Dong TT and Tsim KW: Yu Ping Feng San reverses cisplatin-induced multi-drug resistance in lung cancer cells via regulating drug transporters and p62/TRAF6 signalling. Sci Rep 6: 31926, 2016.

13. Maeda S, Yoshida H, Ogura K, Mitsuno Y, Hirata Y, Yamaji Y, Akanuma M, Shiratori Y and Omata M: H. Pylori activates NF-kappaB through a signaling pathway involving IkappaB kinases, NF-kappaB-inducing kinase, TRAF2, and TRAF6 in gastric cancer cells. Gastroenterology 119: 97-108, 2000.

14. Kong L, Li X, Wang H, He G and Tang A: Calycosin inhibits nasopharyngeal carcinoma cells by influencing EWSAT1 expression to regulate the TRAF6-related pathways. Biomed Pharmacother 106: 342-348, 2018.

15. Bilir C, Engin H, Can M, Likhan S, Demirtas D, Kuzu F and Bayraktaroglu T: Increased serum tumor necrosis factor receptor-associated factor- 6 expression in patients with non-metastatic triple-negative breast cancer. Oncol Lett 9: 2819-2824, 2015.

16. Rezaeian AH, Li CF, Wu CY, Zhang X, Delacerda J, You MJ, Han F, Cai Z, Jeong YS, Jin G, et al: A hypoxia-responsive TRAF6-ATM-H2AX signalling axis promotes HIF1 $\alpha$ activation, tumorigenesis and metastasis. Nat Cell Biol 19: 38-51, 2017.

17. Shi B, Wang Y and Yin F: MALAT1/miR-124/Capn4 axis regulates proliferation, invasion and EMT in nasopharyngeal carcinoma cells. Cancer Biol Ther 18: 792-800, 2017.

18. Li SL, Gao HL, Lv XK, Hei YR, Li PZ, Zhang JX and Lu N: MicroRNA-124 inhibits cell invasion and epithelial-mesenchymal transition by directly repressing Snail2 in gastric cancer. Eur Rev Med Pharmacol Sci 21: 3389-3396, 2017.

19. Liu Q, Luo D, Cai S, Li Q and Li X: P-TNM staging system for colon cancer: Combination of P-stage and AJCC TNM staging system for improving prognostic prediction and clinical management. Cancer Manag Res 10: 2303-2314, 2018.

20. Chen S, Chen H, Gao S, Qiu S, Zhou H, Yu M and Tu J: Differential expression of plasma microRNA-125b in hepatitis B virus-related liver diseases and diagnostic potential for hepatitis B virus-induced hepatocellular carcinoma. Hepatol Res 47: 312-320, 2017.

21. He Z, Yu L, Luo S, Li M, Li J, Li Q, Sun Y and Wang C: miR-296 inhibits the metastasis and epithelial-mesenchymal transition of colorectal cancer by targeting S100A4. BMC Cancer 17: 140, 2017.

22. Liu W, Zhang Q, Li S, Li L, Ding Z, Qian Q, Fan L and Jiang C: The relationship between colonic macrophages and MicroRNA-128 in the pathogenesis of slow transit constipation. Dig Dis Sci 60: 2304-2315, 2015. 
23. Li JJ, Luo J, Lu JN, Liang XN, Luo YH, Liu YR, Yang J, Ding H, Qin GH, Yang LH, et al: Relationship between TRAF6 and deterioration of HCC: An immunohistochemical and in vitro study. Cancer Cell Int 16: 76, 2016.

24. Hueman MT, Wang H, Yang CQ, Sheng L, Henson DE, Schwartz AM and Chen D: Creating prognostic systems for cancer patients: A demonstration using breast cancer. Cancer Med 7: 3611-3621, 2018

25. Wang L, Dou X, Liu T, Lu W, Ma Y and Yang Y: Tumor size and lymph node metastasis are prognostic markers of small cell lung cancer in a Chinese population. Medicine (Baltimore) 97: e11712, 2018

26. Moody L, He H, Pan YX and Chen H: Methods and novel technology for microRNA quantification in colorectal cancer screening. Clin Epigenetics 9: 119, 2017.

27. Pecqueux M, Liebetrau I, Werft W, Dienemann $H$, Muley T, Pfannschmidt J, Müssle B, Rahbari N, Schölch S, Büchler MW, et al: A Comprehensive MicroRNA expression profile of liver and lung metastases of colorectal cancer with their corresponding host tissue and its prognostic impact on survival. Int J Mol Sci 17: pii: E1755, 2016.

28. Zhang J and Ma L: MicroRNA control of epithelial-mesenchymal transition and metastasis. Cancer Metastasis Rev 31: 653-662, 2012.

29. Hur K, Toiyama Y, Takahashi M, Balaguer F, Nagasaka T, Koike J, Hemmi H, Koi M, Boland CR and Goel A: MicroRNA-200c modulates epithelial-to-mesenchymal transition (EMT) in human colorectal cancer metastasis. Gut 62: 1315-1326, 2013.

30. Fang Y, Gu X, Li Z, Xiang J and Chen Z: miR-449b inhibits the proliferation of SW1116 colon cancer stem cells through downregulation of CCND1 and E2F3 expression. Oncol Rep 30: 399-406, 2013.

31. Liu X, Duan B, Dong Y, He C, Zhou H, Sheng H, Gao H and Zhang X: MicroRNA-139-3p indicates a poor prognosis of colon cancer. Int J Clin Exp Pathol 7: 8046-8052, 2014.

32. Zhang J, Lu Y, Yue X, Li H, Luo X, Wang Y, Wang K and Wan J: MiR-124 suppresses growth of human colorectal cancer by inhibiting STAT3. PLoS One 8: e70300, 2013.
33. Wang MJ, Li Y, Wang R, Wang C, Yu YY, Yang L, Zhang Y, Zhou B,Zhou ZG and Sun XF: Downregulation of microRNA-124 is an independent prognostic factor in patients with colorectal cancer. Int J Colorectal Dis 28: 183-189, 2013

34. Qiu Z, Guo W, Wang Q, Chen Z, Huang S, Zhao F, Yao M, Zhao Y and He X: MicroRNA-124 reduces the pentose phosphate pathway and proliferation by targeting PRPS1 and RPIA mRNAs in human colorectal cancer cells. Gastroenterology 149: 1587-1598.e11, 2015.

35. Ueno H, Hase K, Hashiguchi Y, Shimazaki H, Yoshii S, Kudo SE, Tanaka M, Akagi Y, Suto T, Nagata S, et al: Novel risk factors for lymph node metastasis in early invasive colorectal cancer: A multi-institution pathology review. J Gastroenterol 49: 1314-1323, 2014

36. Han L, Jiang Y, Han D and Tan W: Hsp27 regulates epithelial mesenchymal transition, metastasis and proliferation in colorectal carcinoma. Oncol Lett 16: 5309-5316, 2018.

37. Guo Q, Zhao Y, Chen J, Hu J, Wang S, Zhang D and Sun Y: BRAF-activated long non-coding RNA contributes to colorectal cancer migration by inducing epithelial-mesenchymal transition. Oncol Lett 8: 869-875, 2014.

38. Yang WL, Wang J, Chan CH, Lee SW, Campos AD, Lamothe B, Hur L, Grabiner BC, Lin X, Darnay BG and Lin HK: The E3 ligase TRAF6 regulates Akt ubiquitination and activation. Science 325: 1134-1138, 2009.

39. Zhang X, Wu L, Xiao T, Tang L, Jia X, Guo Y, Zhang J, Li J, He Y, Su J, et al: TRAF6 regulates EGF-induced cell transformation and cSCC malignant phenotype through CD147/EGFR. Oncogenesis 7: 17, 2018.

40. Han F, Zhang L, Qiu W and Yi X: TRAF6 promotes the invasion and metastasis and predicts a poor prognosis in gastric cancer. Pathol Res Pract 212: 31-37, 2016.

41. Sun H, Li X, Fan L, Wu G, Li M and Fang J: TRAF6 is upregulated in colon cancer and promotes proliferation of colon cancer cells. Int J Biochem Cell Biol 53: 195-201, 2014.

(i) (2) This work is licensed under a Creative Commons CY NG ND Attribution-NonCommercial-NoDerivatives 4.0 International (CC BY-NC-ND 4.0) License. 